\title{
EchoGéo
}

$21 \mid 2012$

Pays émergents

\section{Les effets de l'émergence sur les discours officiels de promotion de la modernisation en Chine}

Léo Kloeckner

\section{OpenEdition}

Journals

Édition électronique

URL : https://journals.openedition.org/echogeo/13158

DOI : 10.4000/echogeo.13158

ISSN : 1963-1197

Éditeur

Pôle de recherche pour l'organisation et la diffusion de l'information géographique (CNRS UMR 8586)

Référence électronique

Léo Kloeckner, «Les effets de l'émergence sur les discours officiels de promotion de la modernisation en Chine », EchoGéo [En ligne], 21 | 2012, mis en ligne le 10 octobre 2012, consulté le 10 août 2021.

URL : http://journals.openedition.org/echogeo/13158; DOI : https://doi.org/10.4000/echogeo.13158

Ce document a été généré automatiquement le 10 août 2021.

EchoGéo est mis à disposition selon les termes de la licence Creative Commons Attribution - Pas d'Utilisation Commerciale - Pas de Modification 4.0 International (CC BY-NC-ND) 


\title{
Les effets de l'émergence sur les discours officiels de promotion de la modernisation en Chine
}

\author{
Léo Kloeckner
}

Le développement économique rapide de la Chine et son intégration dans les circuits de la globalisation s'accompagnent de changements politiques, sociaux et culturels majeurs, qui marquent le territoire chinois à toutes les échelles. Les sciences sociales désignent ce double processus d'affirmation internationale et de bouleversements internes comme relevant de l'émergence. L'Exposition Universelle qui a eu lieu à Shanghai du $1^{\text {er }}$ mai au 31 octobre 2010 apparaît comme une occasion privilégiée d'étudier ce double processus, puisqu'il se trouve alors concentré dans le temps et dans l'espace, la ville de Shanghai étant soumise à des transformations majeures avant l'ouverture de l'Exposition au public international. L'Exposition elle-même apparaît comme un signe de l'émergence de la Chine au sens de son affirmation sur la scène internationale, en tout cas comme un «signe de l'émergence métropolitaine» de Shanghai, «de son inscription effective dans le réseau des très grandes villes du monde» (Sanjuan, 2009).

J'ai choisi d'interroger le phénomène de l'émergence en analysant le contenu, la présence et la place de l'image dans la ville chinoise, de celle qui couvre ses murs, et qui est produite par les autorités, en m'intéressant en particulier au paysage visuel shanghaien à l'approche de l'Exposition universelle. Par image j'entends une représentation visuelle se trouvant sur un support matériel, mais je m'intéresse aussi à l'image en ce qu'elle a à voir avec le langage, au slogan qui l'accompagne presque systématiquement dans la ville chinoise. Les murs de Shanghai, à l'approche de l'Exposition de 2010, ont en effet vu proliférer un discours faisant la promotion de l'Exposition Universelle et des projets de modernisation de l'espace métropolitain et de normalisation des pratiques urbaines et sociales l'accompagnant. Ces politiques de modernisation ne sont pas nouvelles, et l'Exposition a eu davantage un rôle d'accélérateur que de déclencheur des processus de modernisation urbaine (Sanjuan, 2009), puisque c'est le schéma directeur 1999-2020 qui pilote l'aménagement de la 
municipalité sur le long terme (Henriot, 2011), et que les campagnes de «civilisation spirituelle " (jinsheng wenming) existent depuis 1979 (Brady, 2007). Même s'il n'est pas nouveau, le discours officiel assurant la promotion de ces mesures de modernisation s'est fait plus présent sur les murs de la ville à l'occasion de l'Exposition.

Parmi la grande variété d'images qui couvrent les murs de Shanghai (publicité, petites annonces privées, images de propagande), j'ai choisi de concentrer mon analyse sur celles qui relèvent de ces campagnes de " civilisation spirituelle ». Le corpus d'images, sur lequel s'appuie cet article, se compose de photographies prises lors d'un terrain réalisé entre février et mai 2009 à Shanghai. C'est le souci de rendre compte de la ville chinoise dans sa diversité qui a présidé au choix de ces dernières. Les images proposées proviennent d'espaces publics et résidentiels aux profils socio-économiques très différents. Le quartier de Yuanhenong est habité principalement par des migrants, dont les ressources sociales et économiques sont moindres que celles des habitants du lilong de Ke Ji Tang Fan. Les comités de résidents y jouent de ce fait un rôle différent, comme en témoignent les conditions de production des images choisies.

Les campagnes de « civilisation spirituelle " visent à normaliser les pratiques sociales et les mœurs urbaines, et à impliquer idéologiquement la population dans le projet global de modernisation de la société et de développement économique. Par modernisation j'entends un ensemble de transformations affectant par exemple l'espace, les structures économiques ou les pratiques sociales, souhaitées et pilotées par des acteurs identifiables, le plus souvent dotés d'une légitimité politique, en l'occurrence l'État chinois. Ces transformations se font par référence à une norme, à un étalon prédéfini par le ou les acteurs de la modernisation, et qui varient en fonction du contexte historique et culturel dans lequel ces derniers s'inscrivent. Entendu ainsi le processus de modernisation renvoie à une démarche planificatrice, qui se manifeste par exemple en termes d'aménagement urbain par l'adoption du schéma directeur pour la période 1999-2020. Ces transformations visent à parvenir à une étape, un état ultime, en l'occurrence, puisque l'on s'intéresse à la société urbaine, l'avènement d'une "société harmonieuse" (hexie shehui), dont le projet est formulé en 2003. La perspective moderniste de l'État chinois est relativement classique de la part d'un pouvoir communiste. En ce qui concerne les images auxquelles je m'intéresse ici, elles font la promotion du projet de "civilisation spirituelle", un projet de modernisation des pratiques sociales sur lequel je reviendrai, et je m'attacherai à interroger la place de ce discours visuel dans la ville émergente, ainsi que les référents de ce discours moderniste. La référence y est souvent faite à une urbanité et une citadinité métropolitaines et globalisées. C'est pourquoi on peut interroger la notion d'émergence à travers le discours visuel shanghaien, sa situation et les conditions de sa production.

Parce qu'elle renvoie à un ensemble de processus, de transformations d'ordre politique, social et culturel, la notion d'émergence entre en écho avec les changements impliqués par une politique de modernisation. Pour autant, parler d'émergence, c'est se placer sur un autre plan de l'analyse, puisque la notion n'identifie pas de projet, de volonté politique, et ne renvoie pas forcément à des processus pilotés, planifiés comme c'est le cas lorsque l'on parle de modernisation. A cet égard la notion d'émergence semble plus englobante, parce que plus indéterminée, et renvoie à un contexte global, quand la modernisation renvoie à un ou des projets particuliers. Autrement dit, certaines des transformations économiques, politiques, sociales et culturelles qui caractérisent l'émergence ne sont pas liées nécessairement à un projet politique, ou à l'action d'acteurs publics. Pour autant l'émergence n'est pas à mettre au compte d'un retrait de 
l'État, il s'agit plutôt d'une autre perspective analytique. Certaines politiques de modernisation peuvent ainsi participer de l'émergence, et j'interroge ici la relation qu'entretient le discours officiel de promotion de «civilisation spirituelle » du corps social chinois, avec le contexte général qui est celui de l'émergence. Ces campagnes visuelles font parfois référence à une modernité et des normes sociales particulières, liées au contexte de l'intégration accélérée aux circuits de la globalisation à l'occasion de l'Exposition universelle. Dans quelle mesure le contexte de l'émergence informe-t-il le discours des autorités chinoises sur la modernisation de la ville et du corps social?

Afin de répondre à cette question, il convient de revenir sur les modalités de la présence dans l'espace public chinois, d'un discours visuel officiel de promotion des politiques de modernisation. Le contenu de ce discours intègre des références qui semblent tenir au contexte de l'émergence, ce qui est particulièrement sensible si l'on s'attarde sur le discours ciblant la normalisation des pratiques et des conduites sociales des citadins d'une ville "moderne». Enfin, la façon dont ce discours sur la modernisation s'intègre dans l'espace urbain shanghaien est révélatrice du fonctionnement de la ville chinoise, de ses particularités, mais aussi des recompositions dont elle est le théâtre dans un contexte d'intégration poussée à la globalisation, soit d'émergence.

\section{Propagande politique et espace public}

La prolifération sur les murs de la ville, d'un discours officiel de promotion de la modernisation de la société shanghaienne à l'approche de l'Exposition universelle, est liée au contexte politique autoritaire et au fort contrôle exercé par les autorités chinoises sur l'espace public. Cette présence de la parole politique dans l'espace public chinois n'est pas nouvelle. La pratique de l'affichage ou l'exposition des textes de loi dans les espaces publics urbains est ainsi attestée en Chine depuis le V $\mathrm{V}^{\mathrm{e}}$ siècle avant notre ère. Elle n'a pas à voir seulement avec la nature autoritaire du pouvoir en place, mais aussi avec la dimension performative de l'écriture chinoise, les caractères donnant à la loi ou au slogan une existence qui excède le symbolique (Alleton, 2008). Au point que Roland Barthes, frappé par ses calligraphies rouges suspendues au dessus des bâtiments publics ou des monuments historiques, désigne Mao comme un " logothète ", " un fondateur de langue ", « le logothète remplaçant le nomothète ", la calligraphie se substituant à la loi (Barthes, 2009, p. 152-153).

Aujourd'hui encore «la publicité des lois est toujours la règle» (Alleton, 2008, p. 83). Depuis la mise en place de la politique d'ouverture, traduite notamment par une relative libéralisation économique, le contrôle exercé par les autorités politiques sur l'espace public et sur l'espace visuel urbain s'est renforcé, particulièrement depuis la répression des mouvements de contestation de 1989, lors de laquelle le Département de la propagande a été doté de nouveaux moyens. Le système de propagande chinois est en effet extrêmement ramifié, ce qui permet une diffusion de l'idéologie officielle du sommet à la base, cette dernière étant relayée - davantage qu'élaborée - par les Bureaux de propagande locaux, qui constituent les instances idéologiques de base du régime. On trouve des bureaux de propagande à tous les échelons d'administration du territoire chinois, de l'échelle municipale (dont on trouve un exemple de production dans l'illustration 1) à celle du comité de résidents (jumin weiyuanhui) (voir les illustrations 2 à 6). Les comités de résidents constituent l'échelon de base 
d'administration de la société chinoise, et se superposent à des unités résidentielles collectives. Ils ont pour fonction de faire le lien entre les autorités et les habitants, essentiellement selon une logique descendante. Ils sont notamment chargés du maintien de la sécurité et de l'hygiène publique, mais aussi de la diffusion des politiques nationales et locales (Thireau, 2006). Les Bureaux de propagande des comités de résidents produisent certaines des images qu'ils affichent, mais ils en reçoivent aussi des organes de propagande municipaux ou du district urbain correspondant. Ces instances de relais de l'idéologie officielle se confondent au niveau local avec les «Bureaux de civilisation spirituelle » (Brady, 2007, p. 25).

Les images auxquelles je m'intéresse ici relèvent des campagnes de "civilisation spirituelle ». La notion de "civilisation spirituelle» a été introduite en 1979 par Ye Jianying, qui était alors ministre de la défense (Brady, 2007). Pour autant, elle n'est au fond qu'une nouvelle formulation du projet ancien de modernisation du corps social et des comportements porté par l'État chinois moderne. La "civilisation spirituelle " succède en effet à "l'éducation politique » (zhengzhi jiaoyu) maoïste (Brady, 2007), et joue encore un rôle d'éducation civique et de mobilisation collective (Landsberger, 2008). Les campagnes de " civilisation spirituelle " participent du projet officiel chinois de construire une "société harmonieuse " (hexie shehui). Cette notion, présentée pour la première fois lors de la cinquième session plénière du XVI ${ }^{e}$ Comité central du Parti communiste chinois, fait référence à un ordre socio-économique dans lequel tous les Chinois coexisteraient dans la paix et l'harmonie. Le projet de société harmonieuse renvoie au souci de prendre en compte les disparités de développement liées au processus d'émergence et à l'ouverture économique. Le renouvellement des slogans et de la ligne idéologique officielle illustre les transformations liées au contexte d'émergence en même temps qu'il participe de la volonté des autorités d'encadrer ces dernières.

Le terme de «wenming» (traduit ici par «civilisation») relayé par la propagande officielle renvoie à l'idée de modernité, mais aussi à celle d'adoption d'un comportement « civil », urbain dans les espaces publics. Les campagnes de « civilisation spirituelle" se focalisent en effet, depuis la consécration du slogan en 1996, sur l'hygiène, les bonnes manières et le comportement en public (Brady, 2007), et relèvent d'un projet de « civilisation des mœurs » (Elias, 1991). A la différence d'une propagande classique faisant par exemple la promotion des forces de défense nationales, la "civilisation spirituelle " concerne depuis le début des années 1980 les règles de la circulation en ville (illustration 1), l'hygiène dans les espaces résidentiels collectifs (illustrations 3 et 4 ), ou les comportements publics (illustrations 4 et 5). 
Illustration 1 - Panneau édité par le «Bureau de construction de la civilisation spirituelle de Shanghai ", échelon municipal de gestion des campagnes de civilisation spirituelle.

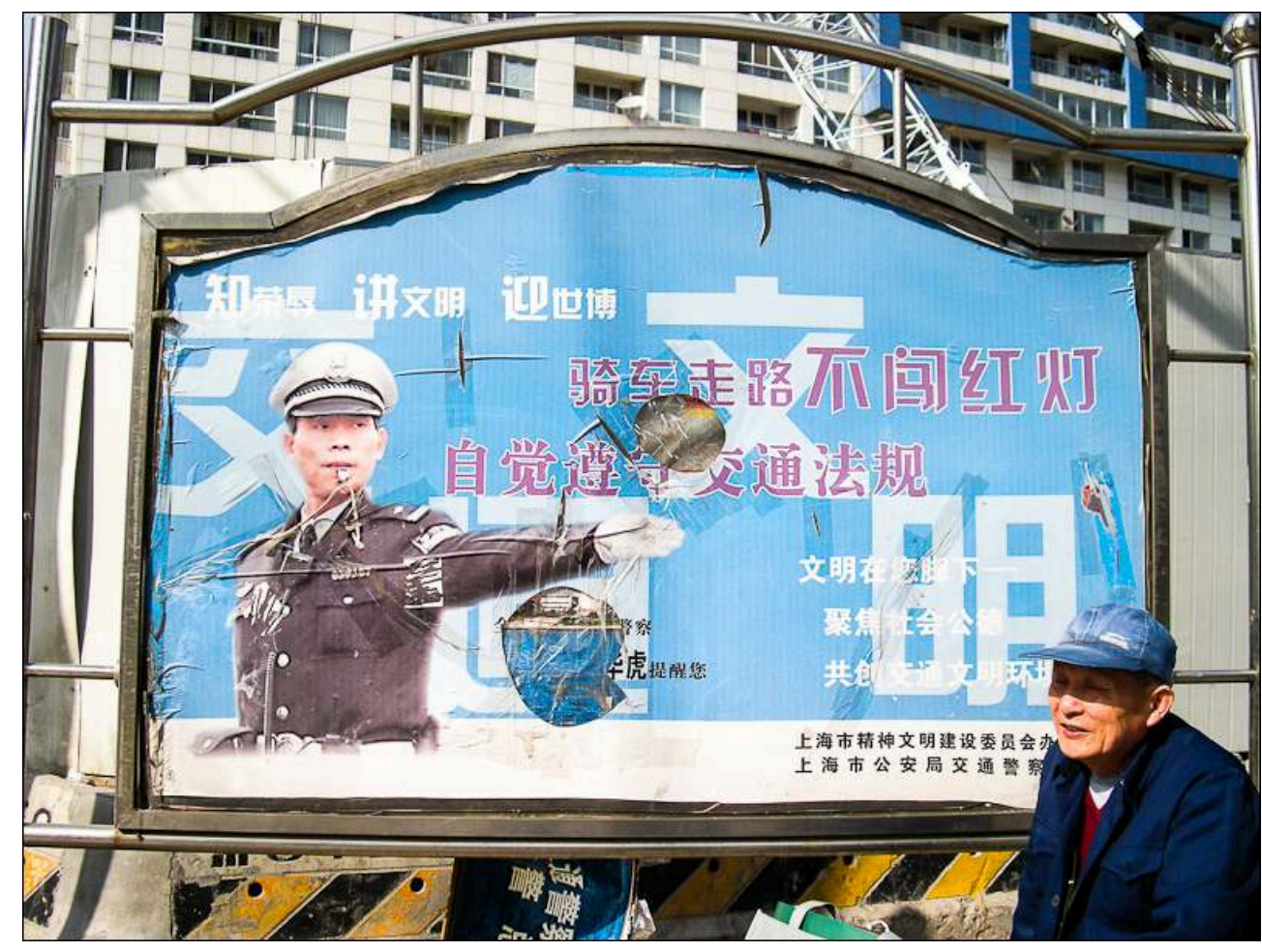

Le rappel de simples règles de circulations : «s'arrêter au feu rouge », « obéir au code de la route consciencieusement ») se double d'obligations morales et d'enjeux civilisationnels : « la civilisation passe par les piétons ; «il faut se concentrer sur la moralité sociale (pour) créer les conditions d'une circulation civilisée ».

Auteur : L. Kloeckner.

Pour Anne-Marie Brady, "le but est de construire un modèle de valeurs neuves et modernes pour la société chinoise. La civilisation spirituelle est une forme de contrôle social, renforcé par la loi et le système policier » (Brady, 2008, p. 25).

Ces campagnes relèvent bien de la modernisation telle que je l'ai définie et traduisent la volonté du pouvoir de transformer le corps social. Même si elles relèvent du discours, et si leur impact est délicat à mesurer, l'implantation des images dans l'espace urbain répond à une stratégie d'efficacité. C'est au seuil des espaces résidentiels administrés par un comité de résidents, que le discours officiel de normalisation des comportements individuels et de "civilisation des mœurs" s'observe de façon privilégiée. Ces espaces sont des lieux de la maîtrise de la séparation entre espace privé et espace public, et de l'élaboration d'une certaine civilité. Le «contrôle social » dont parle Anne-Marie Brady y est d'autant plus fort que l'accès aux espaces résidentiels collectifs, à Shanghai comme en Chine en général, est limité à des points de passage précis, presque toujours placés sous surveillance, les habitants étant par conséquent inévitablement témoins de ces campagnes visuelles.

A l'occasion de l'Exposition universelle, on observe une collusion entre campagnes de civilisation spirituelle et promotion de l'Exposition. Le panneau de civilisation spirituelle situé à l'entrée du quartier de Ke Ji Tang Fan (illustration 2) semble justifier les interdits énoncés par les affichettes (illustration 3), en faisant référence à l'approche de l'Exposition universelle. C'est pourquoi la référence à l'émergence dans le discours chinois de modernisation, de civilisation des mœurs des urbains est 
problématique, l'Exposition Universelle étant perçue par le public chinois lui-même comme un signe de l'émergence, un gage supplémentaire de l'insertion dans le club des grandes puissances mondiales. Dans l'article du Quotidien du Peuple du 4 décembre 2002, consacré à la sélection de Shanghai comme ville hôte pour héberger l'Exposition Universelle de 2010, on pouvait lire: "L'accueil de l'Exposition Universelle en 2010 à Shanghai est une opportunité importante pour la Chine à l'orée d'un nouveau siècle, qui permettra de faire avancer puissamment le développement global de la société et d'accélérer la modernisation socialiste. Nous pensons que la tenue de ces activités internationales de premier plan contribuera positivement à la création d'une nouvelle conjoncture pour le socialisme de marché " (Zhongwei et Chao, 2008). Un tel événement joue de fait un rôle d'accélérateur dans les processus de transformation urbaine puisqu'ils sont pour ainsi dire concentrés en un temps très court par rapport à l'échelle du temps, plus long, de la ville. La bonne tenue de l'événement sert de prétexte aux autorités pour accélérer le train des modernisations, comme l'a montré Anne-Marie Broudehoux pour Pékin à l'occasion des Jeux Olympiques (Broudehoux, 2004).

\section{Faire la promotion de la modernisation au nom de l'émergence?}

C'est parce qu'elle joue un rôle de vitrine du développement économique et urbain chinois que l'Exposition universelle a des effets sur le discours de civilisation spirituelle. Il s'agit bien d'un événement médiatisé de façon internationale, au cours duquel la ville, et indirectement la société chinoise toute entière, sont exposées au regard des visiteurs (on en a dénombré plus de 70 millions pendant toute la durée de l'Exposition) et des téléspectateurs du monde entier. Il s'agit dès lors de ne pas " perdre la face » (diulian) devant ces derniers (Broudehoux, 2004), et de présenter au monde un visage moderne, ce qui passe par la production d'une ville "propre ", débarrassée de ce qui fait figure d'oripeaux du sous-développement, à l'occasion de campagnes d' « embellissement social » (Broudehoux, 2004, p. 174) et de renouvellement urbain. Le contexte de l'émergence influence ainsi en partie le discours de modernisation des pratiques sociales et des comportements citadins.

La modernité à laquelle font référence les campagnes visuelles de civilisation spirituelle est celle d'une métropole globalisée. Le terme même de "wenming " évoque autant le processus de civilisation que l'idée de "modernité » ou d' "occidentalisation », et selon Anne-Marie Broudehoux, renvoie aussi à l'idée « d'être plus avancé, et pas seulement moderne, et dénote un état de civilité étroitement associé avec les cultures industrielles développées d'Asie et d'Occident» (Broudehoux, 2004, p. 176). Ainsi les campagnes de civilisation spirituelle font référence à des normes sociales calquées sur les «normes de distinction sociale des classes supérieures occidentales» (Broudehoux, 2004, p. 177). 
Illustration 2 - Confusion entre campagnes de civilisation spirituelle et promotion de l'Exposition

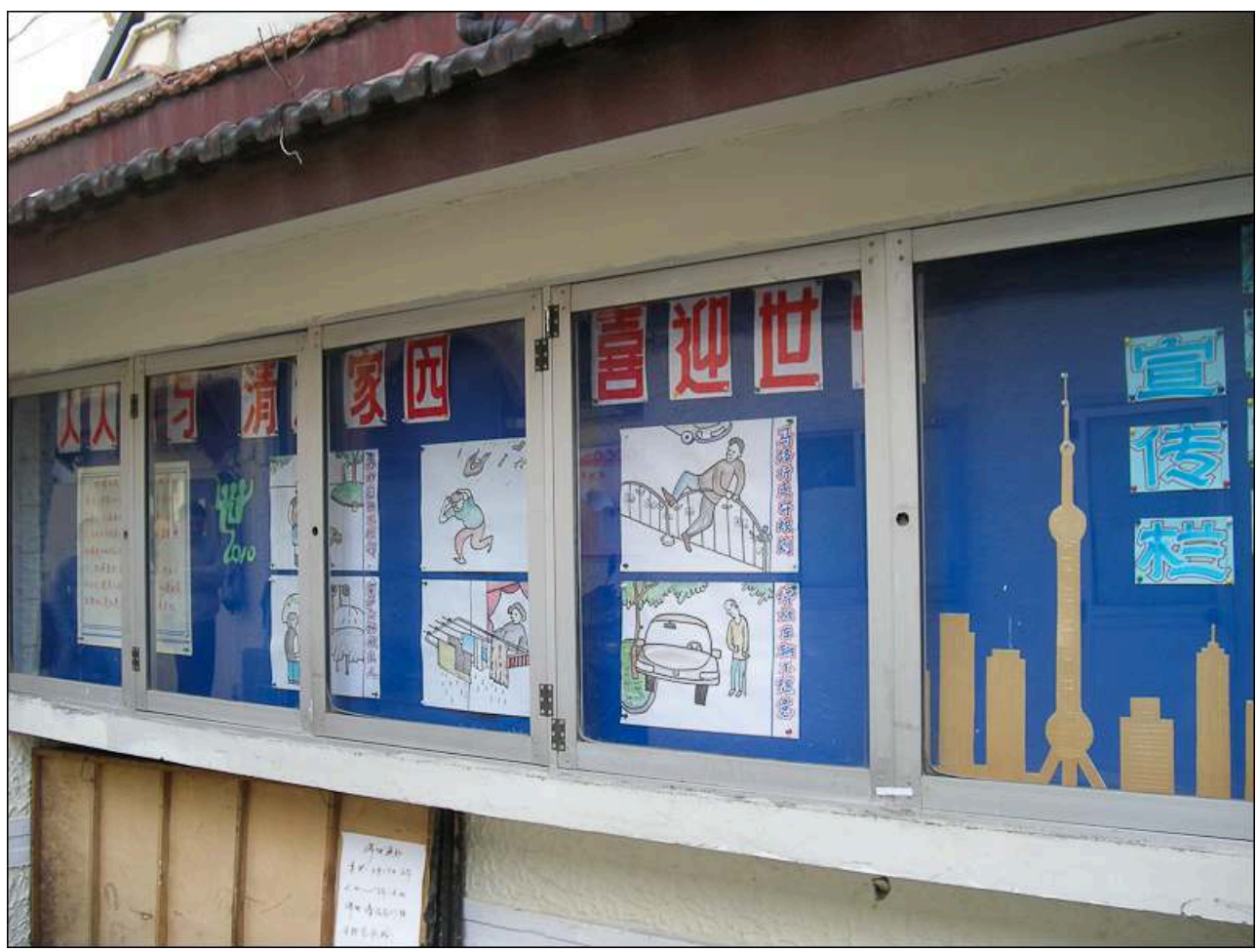

Le panneau de civilisation spirituelle situé à l'entrée du quartier de Ke Ji Tang Fan donne comme justification aux interdits énoncés par les affichettes l'accueil de l'Exposition. On lit en rouge,

" accueillir l'Exposition universelle avec joie ", au-dessus des affichettes au côté desquelles on reconnaît en papier doré la skyline de Pudong, symbole de la modernité métropolitaine shanghaienne. Auteur : L. Kloeckner.

A l'entrée du quartier de Ke Ji Tang Fan, un panneau d'affichage (illustration 2) présente en effet aux résidents des « affiches destinées à la civilisation spirituelle » de la communauté (en bleu au dessus de la skyline de Pudong), et les encourage (en rouge) à «accueillir l'Exposition universelle avec joie». S'ensuit une série de 6 images dessinées à la main qui sont autant de règles de bonne conduite en collectivité, à l'instar de celle que l'on peut voir sur l'illustration 3, «Ne rien jeter des étages, faire attention à la vue lorsqu'il faut étendre le linge ». 
Illustration 3 - Des règles pour une bonne conduite en collectivité

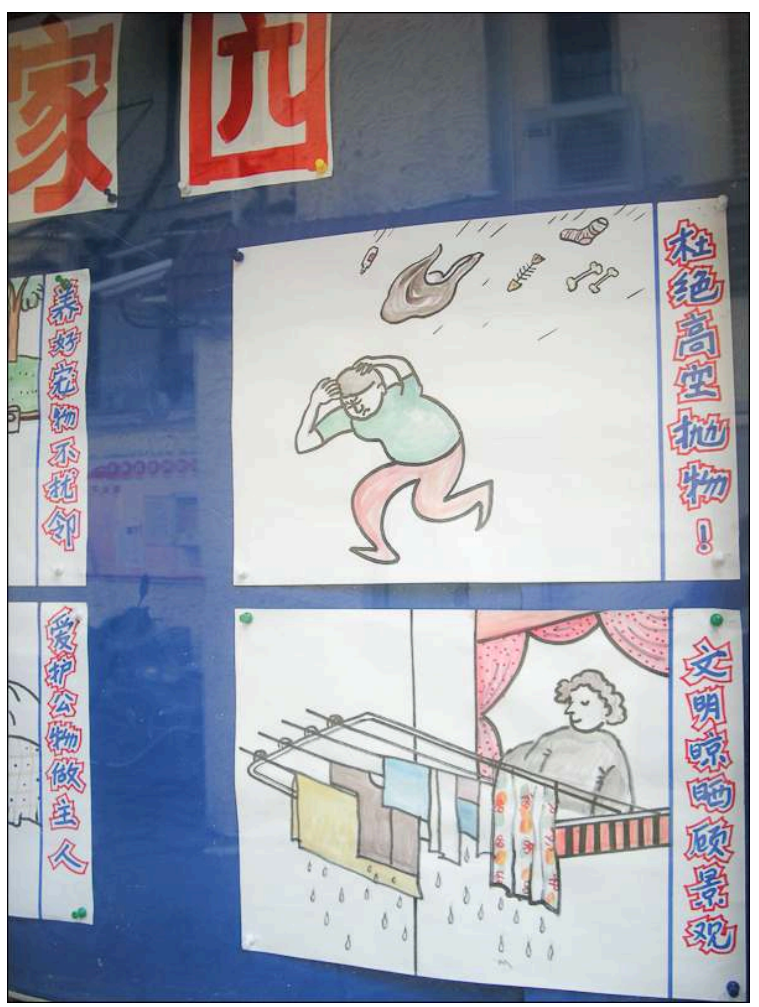

II s'agit de mesures d'ordre hygiénique, mais aussi esthétique, qui s'inscrivent en rupture avec l'ambiance populaire traditionnelle des lilongs shanghaiens (Ged, 2000), dont les ruelles sont souvent envahies de linge qui sèche. « Ne rien jeter des étages, faire attention à la vue lorsqu'il faut étendre le linge ».

Auteur : L. Kloeckner.

Les autres images recommandent de «respecter le code de la route, de se garer correctement", "d'empêcher les animaux domestiques d'être trop bruyants, et de surveiller les affaires d'autrui comme si on en était propriétaire ». Cette campagne se retrouve dans de très nombreux espaces résidentiels collectifs, et s'adresse aux habitants en les interpellant sur leur usage des espaces communs.

Le même bureau de civilisation spirituelle diffuse aussi une campagne qui concerne les comportements des habitants une fois qu'ils sont sortis de l'espace résidentiel communautaire (illustrations 4 et 5). Il n'est pas anodin que cette campagne soit produite par la municipalité de Shanghai. C'est à une citadinité métropolitaine qu'elle fait appel plus qu'à des processus d'appartenance locale au quartier, comme c'est le cas par exemple dans l'appel à protéger du vol les affaires d'autrui. La campagne est précisément menée dans le contexte de l'Exposition Universelle (chaque affiche est surmontée de l'en-tête «Une Exposition splendide, agir avec civilité ») et formule des interdits, stigmatise certaines pratiques au nom de la « civilisation ». L'image la plus emblématique est certainement celle de l'illustration 4 qui condamne la pratique très chinoise du crachat: «commencer par cracher par terre de façon appropriée » (sous entendu, pas n'importe où). 
Illustration 4 - Réformer l'urbanité métropolitaine : « agir avec civilité »

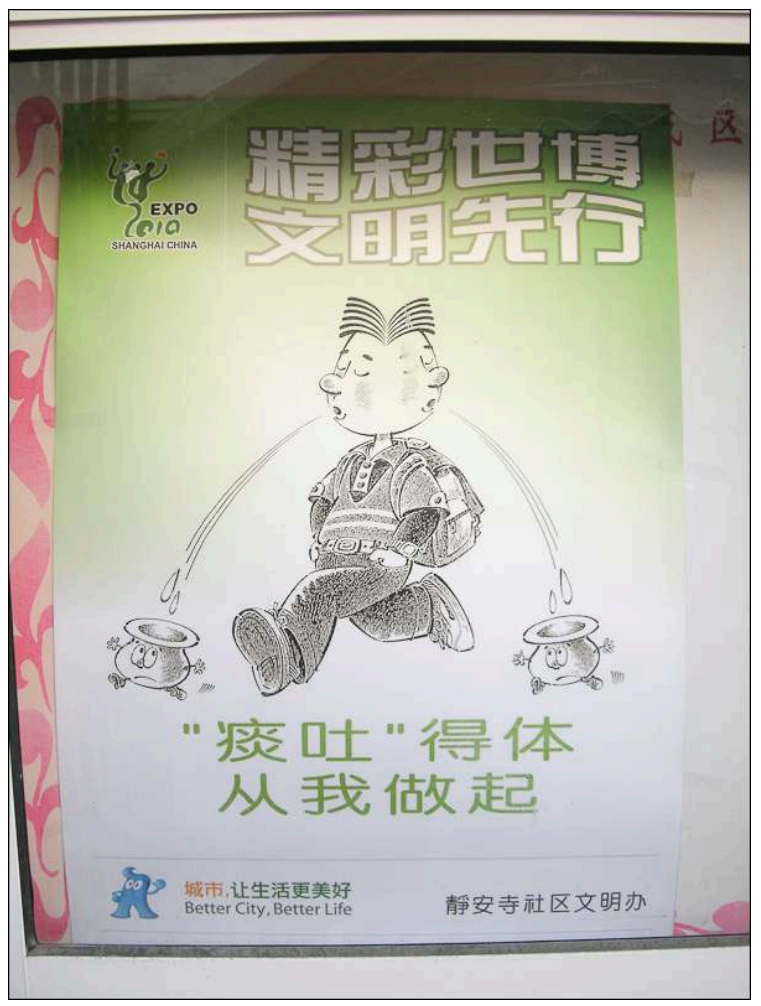

La campagne est menée dans le contexte de l'Exposition Universelle (chaque affiche est surmontée de l'en-tête « Une Exposition splendide, agir avec civilité ») et formule des interdits, stigmatise certaines pratiques au nom de la « civilisation ». Ici, on vise la pratique du crachat : « commencer par cracher par terre de façon appropriée ».

Auteur : L. Kloeckner.

Il s'agit de stigmatiser de façon symptomatique des pratiques qui ne correspondent pas aux codes occidentaux d'urbanité, de pudeur et de civilité, et sont désormais présentées comme des repoussoirs dans la construction de nouvelles normes de citadinité. Pourtant les trois affiches reproduites ici font référence à des pratiques relativement anodines dans l'espace urbain shanghaien, comme dans les villes chinoises en général. Le fait de cracher est perçu d'abord comme un moyen de se purifier d'expectorations jugées malsaines, et non comme une source de souillure de l'espace public. L'illustration 5 fait référence à la façon de s'habiller pour sortir de chez soi et au comportement dans l'espace public, et stigmatise les habitants qui sortiraient en pyjama de chez eux et se comporteraient en somme dans la rue comme chez eux, en parlant trop fort par exemple, alors que ces pratiques constituent la manifestation d'une appartenance locale forte, et sont perçues davantage comme une marque de cordialité que d'incivilité. 


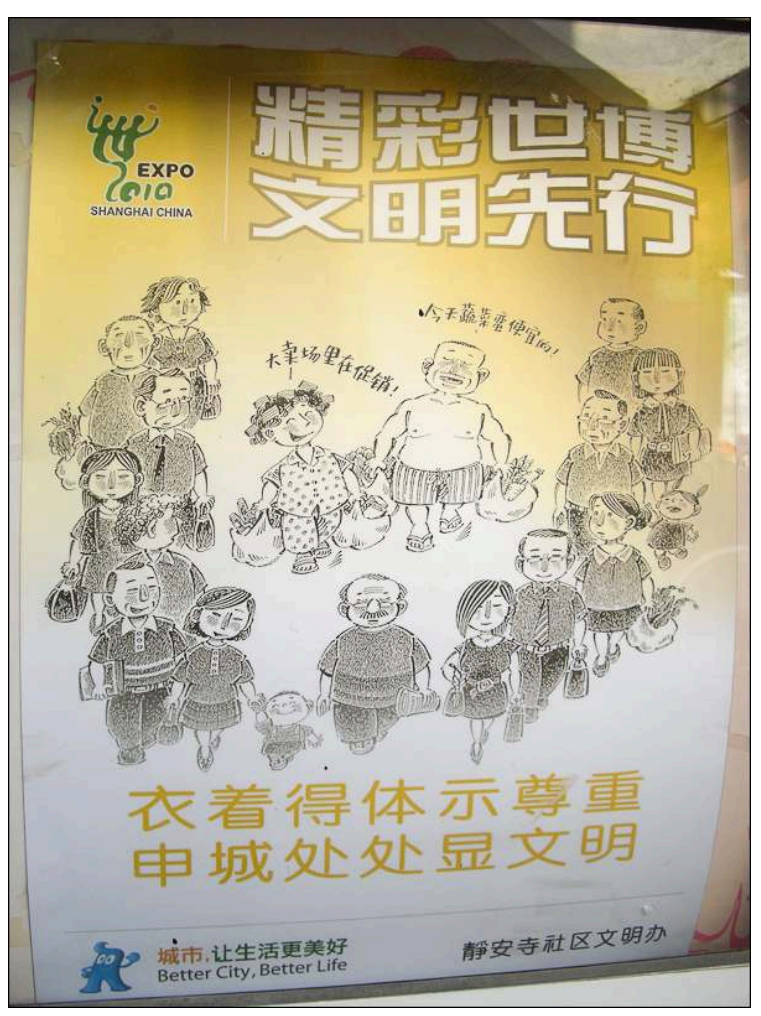

La campagne est menée dans le contexte de l'Exposition Universelle (chaque affiche est surmontée de l'en-tête « Une Exposition splendide, agir avec civilité ») et formule des interdits, stigmatise certaines pratiques au nom de la « civilisation ». «Se vêtir de façon appropriée pour faire preuve de respect », «étendre la civilisation à toute la ville ».

Auteur : L. Kloeckner.

La construction de la norme se joue ici comme en négatif: on s'attache à montrer ce qu'il ne faut pas faire, dans un contexte de sur-médiatisation de la société shanghaienne à l'occasion de l'Exposition Universelle. Le contexte de l'émergence informe donc en partie le discours de modernisation, de civilisation des mœurs produit par les autorités urbaines.

Pour autant on n'identifie pas de discours de l'émergence. C'est seulement la modernité à laquelle fait référence le discours de "civilisation spirituelle», le projet de modernisation des mœurs urbaines, qui est marquée par le contexte de l'émergence. Le terme d'émergence lui-même n'est pas employé par les autorités chinoises. La complexité du terme "wenming » le rend suffisamment malléable pour que le discours de «civilisation spirituelle " puisse adopter une nouvelle modernité de référence, en fonction des impératifs du développement économique et urbain. En cela le projet de modernisation de la société chinoise, promu par les campagnes de civilisation spirituelle, s'inscrit dans un temps plus long que celui du contexte de l'émergence récente, observée depuis les années 1980 . Si l'émergence contamine ce discours visuel, c'est seulement par un effet de contexte. Si l'on peut parler de discours de modernisation sociale, de civilisation des mœurs, on ne peut pas pour autant affirmer l'existence de ce qui correspondrait à un discours de l'émergence.

La grande diversité des conditions d'élaboration des campagnes visuelles de "civilisation spirituelle» met aussi en échec cette idée d'un discours unifié de l'émergence. Certes l'unité de la ligne idéologique est garantie par les instances 
officielles de régulation de la propagande (Brady, 2007), mais la diversité des conditions d'insertion de l'image dans la ville révèle les recompositions économiques et sociales qui la transforment dans un contexte d'émergence.

\section{Des limites de la modernisation du corps social liées à l'émergence}

La variation des contenus du discours officiel de promotion de la «civilisation spirituelle ", et des conditions de leur élaboration, en fonction du contexte urbain dans lequel ils s'inscrivent, rappelle la structure sociale et économique relativement éclatée de la ville chinoise émergente, que Judith Audin compare à une "mosaïque " (Audin, 2008, p. 111). L'émergence semble jouer à cet égard un rôle de limite à l'efficacité des campagnes de civilisation spirituelle, dans la mesure où elle explique en partie cet éclatement d'un discours unique.

\section{La multiplication des acteurs dans la ville socialiste émergente}

C'est particulièrement flagrant si l'on observe le cas du quartier résidentiel populaire de Yuanhenong, habité principalement par des migrants. Le discours de «civilisation spirituelle " que l'on rencontre dans ce quartier, fréquenté par une population de migrants illégaux est révélateur, de par ses conditions de production et de diffusion, des déséquilibres et des disparités qui sont aussi les signes de l'émergence métropolitaine shanghaienne, et d'une polarisation accrue du tissu social. Les migrants chinois en situation d'illégalité ne disposent pas du hukou urbain, le passeport délivré dans le cadre du système d'enregistrement de la résidence et qui donne accès à des services et des avantages particuliers, selon qu'on est enregistré comme urbain ou comme rural (Thireau, 2006). De ce fait, le contrôle spatial exercé par le comité de résidents y est moindre, et son rôle y tient davantage de l'aide sociale que de l'organisation de la vie de la collectivité. Les supports d'affichage sont en effet plus précaires comme en atteste l'illustration 6, puisqu'il s'agit de simples photocopies en noir et blanc, pas d'illustrations plastifiées ou sous verre en couleurs, et que le slogan qui y est inscrit, « Pour accueillir l'expo avec joie, tout le monde nettoie sa maison! », montre bien que la préoccupation première du comité de résidents est l'hygiène, dans un contexte marqué par une forte insalubrité. 


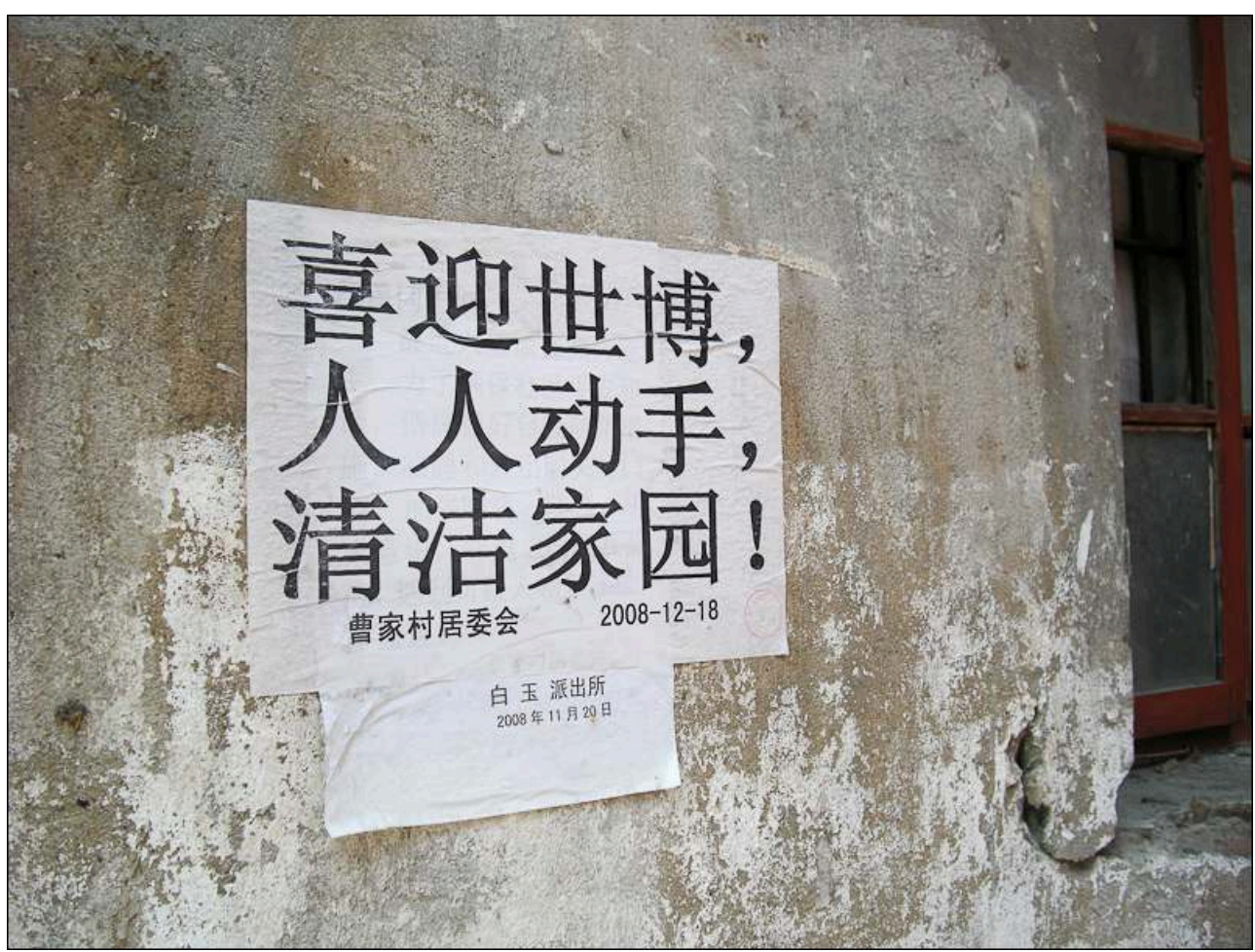

Les moyens du comité de résidents du quartier de la Baiyu Lu sont moindres que dans le cas du quartier de Ke Ji Tang Fan, mais le comité de résidents y a le même souci du respect de l'hygiène: « pour accueillir l'expo avec joie, tout le monde nettoie sa maison! ».

Auteur : L. Kloeckner.

Le comité de résidents n'est pas le seul acteur à produire un discours visuel dans cet espace, les murs sont également occupés par des panonceaux plastifiés en couleurs, qui émanent de la Shanghai Charity Foundation, une fondation philanthropique fondée en 1994 et qui mène des actions sociales à Shanghai. Ce n'est pas un acteur informel puisqu'elle dépend des autorités politiques, mais elle marque la relative impuissance du comité de résidents à contrôler cet espace. Les images qu'elle y publie montrent que si elle porte assistance aux migrants défavorisés économiquement, elle le fait avec la rhétorique propre à la ligne idéologique officielle chinoise, et qu'elle relaie même les slogans officiels. Comme le montrent les illustrations $7 \mathrm{a}$ et $7 \mathrm{~b}$, sur un fond uni ou représentant la ville de Shanghai, se détachent le portrait d'un grand homme, d'une figure célèbre et symbolique pour le pouvoir communiste, ainsi qu'une citation édifiante, qui fait appel à des valeurs morales, à la vertu citoyenne et à une certaine discipline. Sur l'illustration $7 \mathrm{a}$ on observe ainsi à l'entrée du quartier un slogan relativement classique du parti, «Développer les œuvres de bienfaisance et construire un Putuo harmonieux » (Putuo est le nom d'un des districts de Shanghai). 
Illustrations $7 \mathrm{a}$ et $7 \mathrm{~b}$ - De nouveaux relais des campagnes de « civilisation spirituelle »

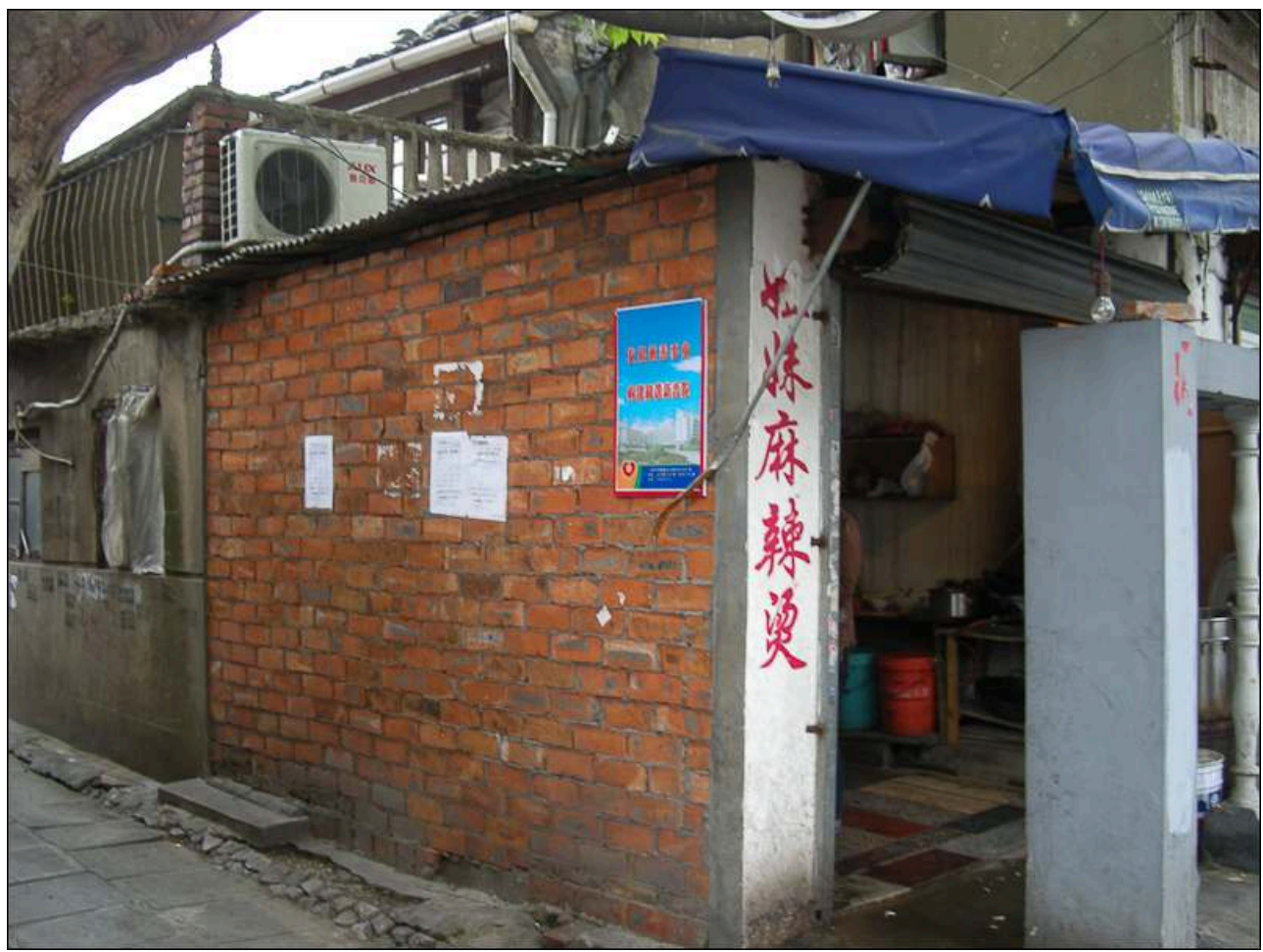

A l'entrée du quartier populaire de la Baiyu Lu, sur les panonceaux produits par la Shanghai Charity Foundation, on peut lire un slogan relativement classique du parti, « Développer les œuvres de bienfaisance et construire un Putuo harmonieux ».

Auteur : L. Kloeckner. 


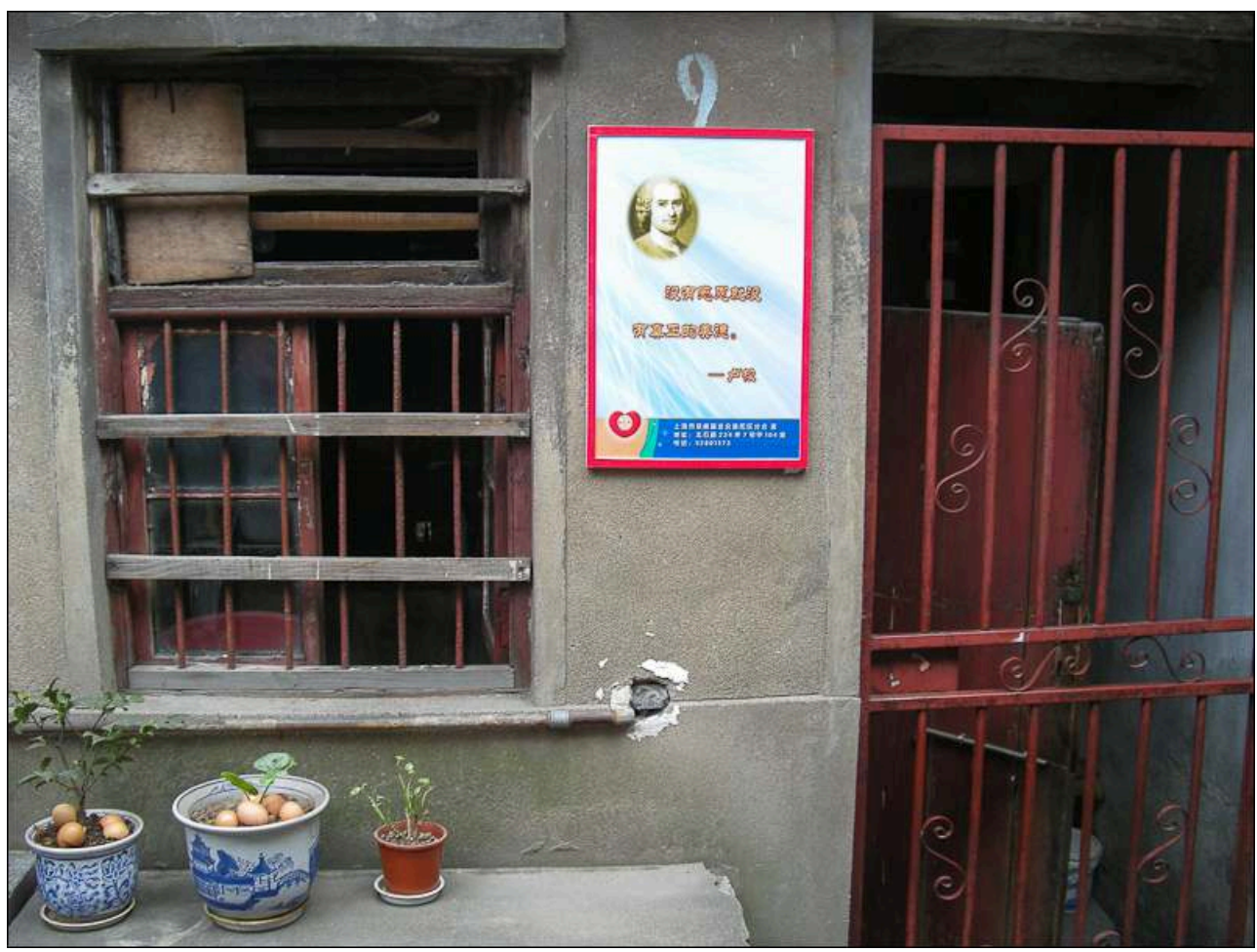

Le discours produit par la Shanghai Charity Foundation a également un rôle de « civilisation des mœurs », appelle en tout cas à une certaine discipline. Une citation de Rousseau assène : « II n'y a pas de vraie vertu sans gratitude».

Auteur : L. Kloeckner.

La teneur davantage moralisatrice du discours adressé aux migrants, par les autorités locales comme par la Shanghai Charity Foundation, témoigne de leur statut particulier dans le corps social urbain. Les migrants illégaux qui composent la majorité de la population du quartier sont en effet perçus comme un obstacle au processus de civilisation spirituelle et « d'embellissement social » (Broudehoux, 2004, p. 174) déployé par les autorités. Ils sont en effet systématiquement évacués de l'espace public par les autorités chinoises qui en ont fait le repoussoir de la modernité urbaine chinoise. Au sens figuré, puisqu'ils n'ont pas les mêmes droits que les résidents détenteurs du hukou urbain, comme au sens propre, puisque les travailleurs migrants de l'économie informelle sont la cible des autorités urbaines, chargées par le gouvernement central de faire disparaitre de l'espace public urbain toute forme d'informalité, associée au sous-développement.

L'existence d'un tel quartier témoigne d'un relatif échec des politiques de modernisation urbaine et sociales menées par les autorités, qui se retrouvent débordées par les mobilités migratoires par exemple, et est précisément un signe de l'émergence. Les transformations sociales qui s'y opèrent, en l'occurrence l'intégration de migrants illégaux à la société urbaine shanghaienne (Zhao, 2011) échappent en effet en grande partie au contrôle politique, au projet de modernisation tel que je l'ai défini. La multiplication des acteurs s'y faisant le relais du discours officiel illustre également un des effets de l'émergence sur la gouvernance du secteur de la propagande, sur la façon dont se déroulent la prise de décision et les arbitrages entre différents acteurs 
dans le processus d'élaboration et de diffusion des campagnes de «civilisation spirituelle » dans l'espace urbain.

Le contexte de l'émergence en Chine se caractérise par une certaine instabilité institutionnelle et une recomposition des jeux d'acteurs, notamment en termes de gestion urbaine et administrative. L'ère de réforme et d'ouverture est marquée par une relative libéralisation, et l'apparition d'acteurs économiques privés, qu'ils soient ruraux ou urbains, qui ne signifient pas pour autant un retrait de l'État. On assiste d'une manière générale à une recomposition et un rééquilibrage des capacités d'initiative entre acteurs privés et publics, qui se traduisent souvent par une diminution des capacités d'initiatives financières des acteurs publics.

\section{La privatisation de la propagande}

Une des principales manifestations de la situation de précarité relative dans laquelle se trouvent les acteurs institutionnels publics et officiels, est la mise en place de partenariats publics-privés ou de joint-ventures entre entreprises chinoises et étrangères. Un des signes forts de l'incapacité dans laquelle se trouvent les organes de propagande à contrôler complètement la production et la diffusion d'un discours visuel sur la modernisation de la ville et du corps social, s'observe dans la mise en place de partenariats entre acteurs publics et privés dans ce secteur. C'est d'abord précisément du fait du contrôle exercé par l'État sur la publicité que l'on a observé un transfert de technologies entre le secteur publicitaire et les organes de propagande. Le secteur publicitaire est en effet étroitement contrôlé par l'État depuis la réhabilitation de la publicité en 1979. La State Administration for Industry and Commerce (SAIC), sous l'autorité du Ministère du Commerce Extérieur et de la Coopération Economique, règlemente le secteur publicitaire, la production de la publicité et contrôle son contenu (Colomb, 1997). En octobre 1987 est promulgué le $1^{\text {er }}$ règlement d'administration de la publicité, dont le premier article affirme qu'il faut "promouvoir le développement de l'activité publicitaire et utiliser le média publicitaire pour servir l'édification socialiste " (Colomb, 1997, p. 122). En 1994 paraît un nouveau texte de loi sur la publicité, la « loi sur la publicité », lors de la $8^{\text {ème }}$ Assemblée populaire nationale de Chine. Le contenu de la publicité est soumis à un contrôle étroit : s'il s'agit de promouvoir le «socialisme de marché ", il n'est pas question que celle-ci se fasse le relais de valeurs subversives. L'espace visuel urbain reste lui aussi sous contrôle, la publicité ne s'affiche pas partout. Les annonces publicitaires destinées à être exposées en extérieur sont installées sur accord du gouvernement local. Pour autant, et même si les départements de propagande se sont vu doter de nouveaux moyens après 1989, les instances locales, les Bureaux de Civilisation Spirituelle n'en ont pas moins une capacité d'initiative limitée du fait de leur manque de moyens.

Steven Wayne Lewis désigne la collaboration qui existe entre publicitaires et responsables locaux de la propagande comme une "privatisation des cadres " (Lewis, 2002, p. 143). Elle a eu pour double effet d'assurer le contrôle du secteur publicitaire et de former les cadres des organes de propagande aux nouvelles méthodes de communication. Cela se traduit concrètement dans l'espace visuel urbain par ce qu'il désigne comme des panneaux de propagande "privatisés ", c'est-à-dire comportant à la fois un message politique, et un message publicitaire marchand. On peut en observer un exemple dans le panneau en métal situé devant le commissariat de la rue Jiangsu (illustration 8). 
Illustration 8 - Un exemple de la privatisation de la propagande dite classique

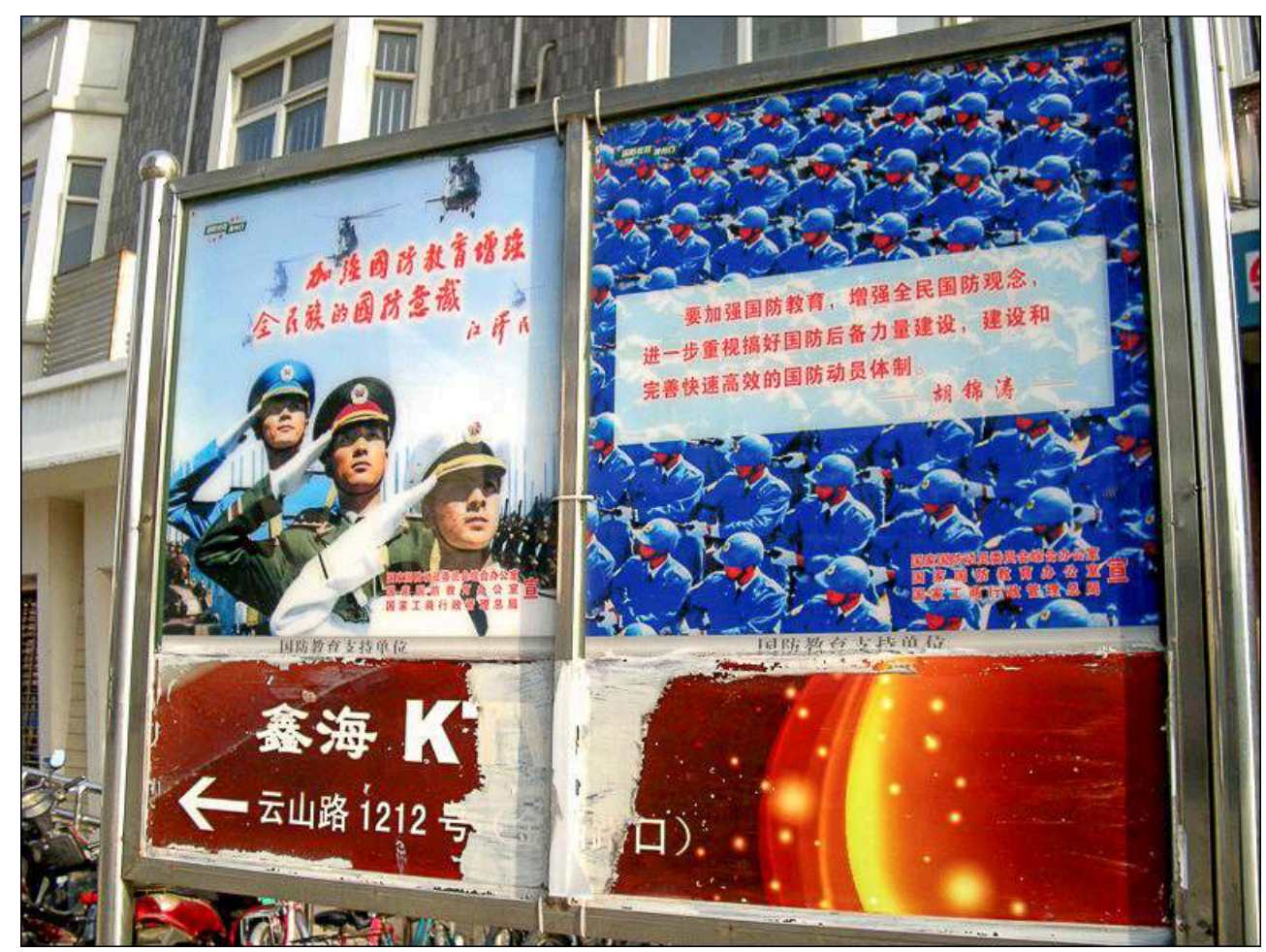

On peut y voir deux affiches surmontant un bandeau publicitaire pour un KTV (l'affiche est partiellement arrachée), une chaîne de karaoké. A gauche, une citation de Jiang Zemin : « Mettre en œuvre l'éducation de la sécurité nationale pour améliorer l'idée de la protection de la population ». A droite, une citation de Hu Jintao : « L'éducation de la sécurité nationale doit être renforcée, le concept de sécurité nationale de tout le peuple doit être amélioré, une attention plus grande doit être portée à la construction d'une force de réserve, pour construire et parfaire un système rapide et efficace de protection nationale ». De façon ironique, la publicité pour le karaoké fait écho à la façon dont sont répétés et scandés les slogans officiels du Parti dans l'espace urbain.

Auteur : L. Kloeckner.

Même dans les espaces résidentiels, dans lesquels le contrôle social et le contrôle de l'espace visuel semblent optimisés, certains signes trahissent le bouleversement des jeux d'acteurs dans un contexte d'émergence. Du fait de leur rôle de relais de la propagande officielle, les comités de résidents locaux en viennent parfois également à diffuser ce que Steven Wayne Lewis désigne comme une propagande "privatisée ", comme en atteste par exemple dans le quartier résidentiel de Ke Ji Tang Fan le panneau édité par le Bureau de Civilisation Spirituelle de Jing'an (illustration 9). 


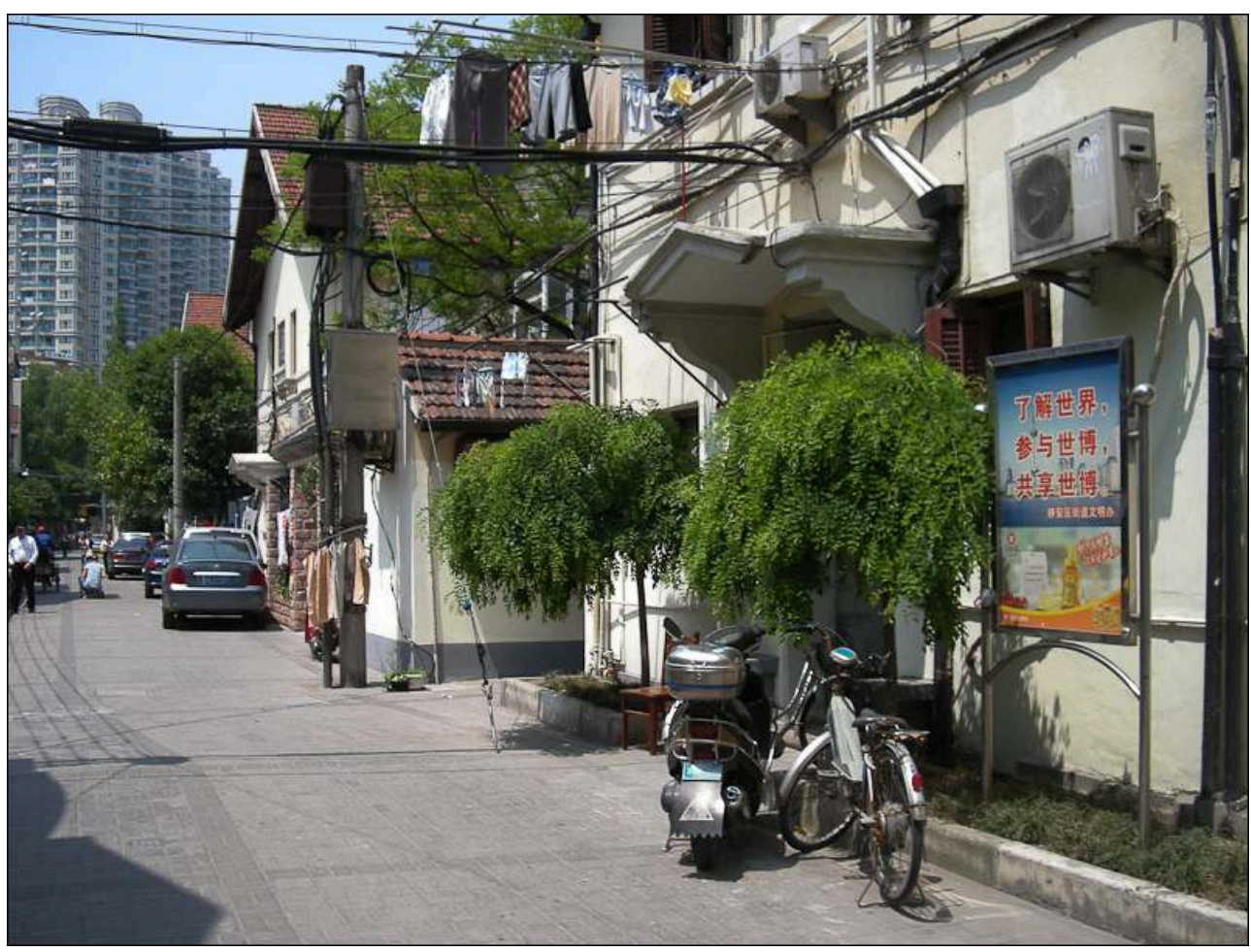

Sur ce panneau en métal un slogan de promotion de l'Exposition universelle (« Comprendre le monde, participer à l'Exposition universelle, profiter de l'Exposition universelle ») sur fond de skyline shanghaienne surmonte une publicité pour du miel.

Auteur : L. Kloeckner.

Ce phénomène de "privatisation » de la propagande peut-être lu non pas tant comme une modernisation de cette dernière, que comme une preuve du manque de moyens auquel doivent faire face les Bureaux de Civilisation spirituelle aux différentes échelles. Le manque de moyens matériels des Bureaux locaux de civilisation spirituelle s'observe également dans la nature des supports auxquels ils ont recours pour diffuser les slogans officiels. Les comités de résidents disposent certes d'espaces d'affichage, mais ces derniers ne sont pas toujours vitrés ni éclairés, et le recours au dessin à la craie sur un tableau noir est très courant (Brady, 2007). Les variations de forme et de support, qui existent entre les images de propagande et la publicité, beaucoup plus voyante (écrans géants, panneaux lumineux), reflètent la relative précarité dans laquelle se retrouvent les acteurs institutionnels publics et officiels dans un contexte d'émergence.

\section{Conclusion}

Les images, qui ont proliféré sur les murs de Shanghai à l'approche de l'Exposition universelle, donnent une certaine évidence aux processus qui transforment la ville chinoise dans un contexte d'émergence. Elles permettent d'interroger ce dernier, puisqu'on lit dans le discours visuel shanghaien de promotion de la «civilisation spirituelle» des traces de ces différents processus, désignés comme relevant de l'émergence. La grande capacité d'adaptation de ce discours est un signe de l'absence d'une stratégie globale à l'échelle de la ville de la part des acteurs officiels, qui a pour conséquence un certain pragmatisme de la part des autorités urbaines dans la diffusion 
de la doctrine officielle. Les variations des conditions d'élaboration des campagnes de civilisation spirituelle, qui poussent parfois les autorités publiques à déléguer leur diffusion à des acteurs privés, ne relevant pas traditionnellement du secteur de la propagande, reflète une certaine précarisation de la marge de manœuvre des autorités locales en contexte d'émergence.

La référence à la notion d'émergence dans le discours de la modernisation n'est par ailleurs qu'indirecte, on ne peut pas désigner ce discours de civilisation, au sens de modernisation, comme un discours de l'émergence. Ce discours fait référence à des processus relevant de l'émergence, convoque et construit des normes qui sont celles d'une urbanité métropolitaine, globalisée et policée. Cela ne reflète pas un souci des autorités chinoises pour l'émergence en elle-même, puisque leur seul projet est bien celui de la modernisation, c'est à dire de l'avènement d'une modernité particulière. Les murs de la ville chinoise jouent en cela un rôle d'écrans, sur lesquels sont projetés, comme de façon incantatoire, les projets d'urbanité des autorités et des acteurs urbains. La notion d'émergence n'en reste pas moins opératoire pour rendre compte de la complexité des conditions de production de ce discours qui couvre les murs de la ville chinoise.

\section{BIBLIOGRAPHIE}

Alleton C., 2008. L'Ecriture chinoise, le défi de la modernité. Paris, Albin Michel, 235 p.

Audin J., 2008. Le quartier, lieu de réinvention des relations Etat-société en Chine urbaine: l'exemple des comités de résidents à Pékin. Raisons politiques, 2008/01, $\mathrm{n}^{\circ} 29$, Presses de Sciences Po, p. 107-117.

Barthes R., 2009. Carnets du voyage en Chine. Carnet 2, vendredi 26 avril 1974, Paris, Christian Bourgois Editeur, $252 \mathrm{p}$.

Brady A-M., 2007. Marketing Dictatorship. London, Rowman \& Littlefield Publishers, 232 p.

Broudehoux A.-M., 2004. The Making and Selling of Post Mao Beijing. Routledge, 280 p.

Colomb D., 1997. L'essor de la communication en Chine : Publicité et télévision au service de l'économie socialiste de marché. Paris, L'Harmattan, collection Champs visuels, 315 p.

Elias N., 1991. La Civilisation des Mœurs. 1939, Paris, Calmann-Lévy, 342 p.

Ged F., 2000. Shanghai. Paris, Institut français d'architecture, 64 p.

Henriot C., 2011. Shanghai, les périphéries urbaines d'une métropole émergente : le cas de la ville nouvelle de Songjiang. Grafigéo, $\mathrm{n}^{\circ}$ 35, Paris, $107 \mathrm{p}$.

Landsberger S., 2008. Chinese Propaganda Posters. Paris, Taschen, 240 p.

Lewis S. W., 2002. What can I do for Shanghai ? Selling Spiritual Civilization in Chinese Cities.In Hemelryk Donald S., Keane M. et Hong Y., 2002,Media in China : consumption, content and crisis, London,Curzon Press, p. 139-151. 
Lewis S. W., 2003. The Media of New Public Spaces in Global Cities, Subway advertising in Beijing, Hong Kong, Shanghai, Taipei. Journal of Media and Culture, vol. 17, n 3, 2003, p. 261-271.

Lewis S. W., 2009. Political and economic implications of new public spaces in Chinese and Asian global cities. In Fulong W., 2009, Globalization and the Chinese City, London, Routledge, p. 190-208.

Piveteau A., Rougier E., 2010. Émergence, l'économie du développement interpellée. Revue de la Régulation, en ligne, $\mathrm{n}^{\circ} 7$, 1er semestre 2010. http://regulation.revues.org/7734?\&id=7734 (accédé le 2 octobre 2012).

Rose G., 2007. Visual Methodologies : An Introduction to the Interpretation of Visual Materials. London, Sage Publications, 345 p.

Sanjuan T. (dir.), 2006. Le Dictionnaire de la Chine Contemporaine. Paris, Armand Colin, 303 p.

Sanjuan T. (dir.), 2007. Atlas de la Chine, les mutations accélérées. Paris, Autrement, 79 p.

Sanjuan T., 2009. Atlas de Shanghai. Paris, Editions autrement, coll. Mégapoles, 84 p.

Sanjuan T., 2010. La Chine et le monde chinois, une géopolitique des territoires. Paris, Armand Colin, $284 \mathrm{p}$.

Thireau I., 2006. Enregistrement de la résidence. In Sanjuan T., Dictionnaire de la Chine Contemporaine, Paris, Armand Colin, p 89-91.

Thireau I., 2006. Résidents (Comité de). In Sanjuan T., Dictionnaire de la Chine Contemporaine, Paris, Armand Colin, p. 221.

Zhongwei W., Chao S., 2008. The World Exposition Reader. Shangahi, Shanghai Scientific and Technological Literature Publishing House, $230 \mathrm{p}$.

Zhao Y., 2011. Construction des espaces urbains et rénovation d'un quartier de Shanghai : la problématique de la migration et du changement social. Shanghai, Shanghai Joint publishing Co, $430 \mathrm{p}$.

\section{RÉSUMÉS}

A l'approche de l'Exposition Universelle de Shanghai, les images des campagnes de « civilisation spirituelle » se multiplient dans les espaces publics et résidentiels. Le contexte de l'émergence joue un rôle de répertoire de normes de la modernité, intégrées au discours de modernisation des mœurs porté par les autorités. La notion d'émergence reste cependant absente du discours officiel, et semble surtout opératoire pour rendre compte des transformations de ses conditions de production. La façon dont il s'intègre dans l'espace urbain shanghaien est révélatrice des recompositions dont la ville chinoise est le théâtre dans un contexte d'intégration poussée à la globalisation.

Before the World Exposition in Shanghai, « spiritual civilization » visual campaigns (propaganda and public advertising images) had spread in publics spaces and residential areas throughout the city. They promote a new civilization of manners and public behaviors, referring to apparently globalized norms. Even though this visual discourse does not refer directly to the notion of emergence, it appears to be useful to explain the transformations in the production process of the official discourse. The way this discourse fits into the shanghainese urban space reveals the transformations that Chinese cities are undergoing in the context of an accelerated integration to globalization. 
INDEX

Mots-clés : civilisation spirituelle, émergence, image, modernisation, Shanghai

Keywords : emergence, image, modernization, Shanghai, spiritual civilization

Thèmes : Sur le Champ - Sur le Terrain

\section{AUTEUR}

\section{LÉO KLOECKNER}

Léo Kloeckner, leo.kloeckner@hotmail.fr, agrégé, est étudiant à l'ENS de Lyon et à l'université Paris 1 en master 2. 